\title{
The fine structures of Cystocaulus ocreatus (Nematoda: Protostrongylidae) and the related lung pathology
}

\author{
K. YILDIZ ${ }^{1, *}$, S. KARAHAN ${ }^{2}$, K. CAVUSOGLU ${ }^{3}$ \\ ${ }^{1}$ Department of Parasitology, Faculty of Veterinary Medicine, Kirikkale University, Department of Parasitology, \\ 71450 Kampus/ Kirikkale, Turkey; E-mail: kyildiz@kku.edu.tr; ${ }^{2}$ Department of Basic Sciences, Faculty of Veterinary \\ Medicine, Kirikkale University; ${ }^{3}$ Department of Biology, Faculty of Science-Art, Kirikkale University
}

\begin{abstract}
Summary
In the present study, the fine surface structures of Cystocaulus ocreatus and its worm as well as brood nodules obtained from the lung of Akkaraman sheep are demonstrated by scanning electron and light microscopy. The mouth of the parasite obtained from the worm nodules is located at the anterior tip and encircled by a collar of tissue. The mouth opening appears as three slits conjoined in the shape of a capital case letter $\mathrm{Y}$, creating three lips. The mouth is surrounded by six papillae just behind the collar. The anterior end was similar in both sexes and covered by numerous wrinkles. Female parasite has a bell-shaped pro-vagina. The posterior end of the female parasite was sharply pointed. The male parasite has a bursa. In the worm nodule, curled parasites were enclosed by a capsula and no eggs and larvae of the parasite were observed. In the brood nodule, heavily affected alveoli and bronchioles contained excessive amounts of larvae and eggs.
\end{abstract}

Key words: Cystocaulus ocreatus; lungworm; worm nodule; brood nodule; scanning electron microscope

\section{Introduction}

Cystocaulus ocreatus is a member of Protostrongylidae family that causes infections in alveoli, alveolar ducts, and bronchi in small ruminants (Anderson, 2000; SattlerováŠtefančíková, 2005; Schnieder, 2000). The resulting lung lesions during different developmental stages of the parasite reduce the lung performance remarkably so that economical losses due to $C$. ocreatus infections can reach to a significant degree. C. ocreatus is highly prevalent among sheep and goat populations worldwide (Hernandez de Lujan et al., 2001; Radfar \& Ghorbani, 2003; Yildiz, 2006). Reports also exist on the presence of Protostrongylidae in ruminants of wildlife, including chamois and moufflons (Meana et al., 1996; Sattlerová-Štefančíková, 2005).

The life cycle of $C$. ocreatus includes five stages of larvae and a mature form (Anderson, 2000). The first stage larvae invade and develop to the second and third larvae stages in a variety of gastropods (Anderson, 2000; Schnieder, 2000). Numerous species of snails are the appropriate intermediate hosts for $C$. ocreatus. Ingested along with the infected snail, larvae in the third stage subsequently reach the lung by way of the hepatic portal system or lymph vessels. The parasite moulted twice in interstitial tissue of the lung migrates to the alveolar duct. $C$. ocreatus becomes mature in the lung by $28-30$ days of ingestion of larvae (Anderson, 2000; Schnieder, 2000). The numerous muscles and contractile elements enable the parasite in its mobility (Guttekova \& Zmoray, 1974). The mature female lays down unembryonated eggs that yield to the first stage larvae (Anderson, 2000; Schnieder, 2000). The first stage larvae is forwarded to the pharynx by cough and swallowed. The first stage larvae, subsequently, excreted through faeces and contaminate the animal surrounding (Anderson, 2000; Schnieder, 2000).

Protostrongylid nematodes including $C$. ocreatus form brood and worm nodules in the lung (Schnieder, 2000). The severity of infection is directly related to the number of these nodules (Berrag \& Cabarret, 1997). Grossly, the worm and brood nodules of $C$. ocreatus can be morphologically differentiated according to their color and shape: the worm nodule is yellow to brown in color and pin-head in shape while brood nodule is green in color and pyramid or semicircular in shape. A worm nodule contains only an immature parasite in general; however, many female and male mature parasites, their eggs, and larvae constitute a brood nodule (Schnieder, 2000).

\footnotetext{
* Corresponding author
} 
Despite the importance of $C$. ocreatus in etiopathogenesis of bronchopneumoni in small ruminants, information on morphology of the adult and larva $C$. ocreatus is limited and our literature review could not find a study illustrating the scanning electron microscopic (SEM) features of $C$. ocreatus. Thus, the objective of the present study was to characterize the SEM characteristics of the female and male $C$. ocreatus as well as the brood and worm nodules containing various stages of $C$. ocreatus.

\section{Material and Methods}

The lung specimens containing brood and worm nodules were collected from Akkaraman sheep naturally infected with $C$. ocreatus. Using a lancet, female and male parasites were recovered from worm nodules. A healthy lung specimen from the same breed was also processed.

For SEM analysis, parasites and lung tissue were fixated in $3 \%$ gluteraldehyde buffered in sodium phosphate (PBS)
$\left(\mathrm{pH} \mathrm{7.2)} \mathrm{for} \mathrm{two} \mathrm{hours} \mathrm{at} 4^{\circ} \mathrm{C}\right.$. Upon rinsed in PBS three times, samples were re-fixated in $1 \%$ osmium tetroxide in PBS for two hours. After fixation, samples were washed in PBS for overnight, then dehydrated in ethanol series (50 $\%, 60 \%, 70 \%, 80 \%, 90 \%, 95 \%$ and $99 \%$ ), and airdried. Specimens were mounted onto stubs by conductive double-sided adhesive tape, sputter coated with a thin layer of gold by Polaron SC-500 (Microtech, East Sussex, England), and viewed on a scanning electron microscope (JSM 5600 JEOL SEM, Jeol Co., Tokyo, Japan).

For histological observation, samples of the lung were fixated in $10 \%$ neutral formalin, dehydrated, embedded in paraffin wax, sectioned on rotary microtome at a thickness of $5 \mu \mathrm{m}$, stained with haematoxylin and eosin, and viewed on a light microscope (BX50 light microscopy (Olympus Optical Co., Ltd., Tokyo, Japan).

The parasites recovered from worm nodules were also evaluated on a light microscope following a single presservation in $70 \%$ ethanol. All worms were identified accord-

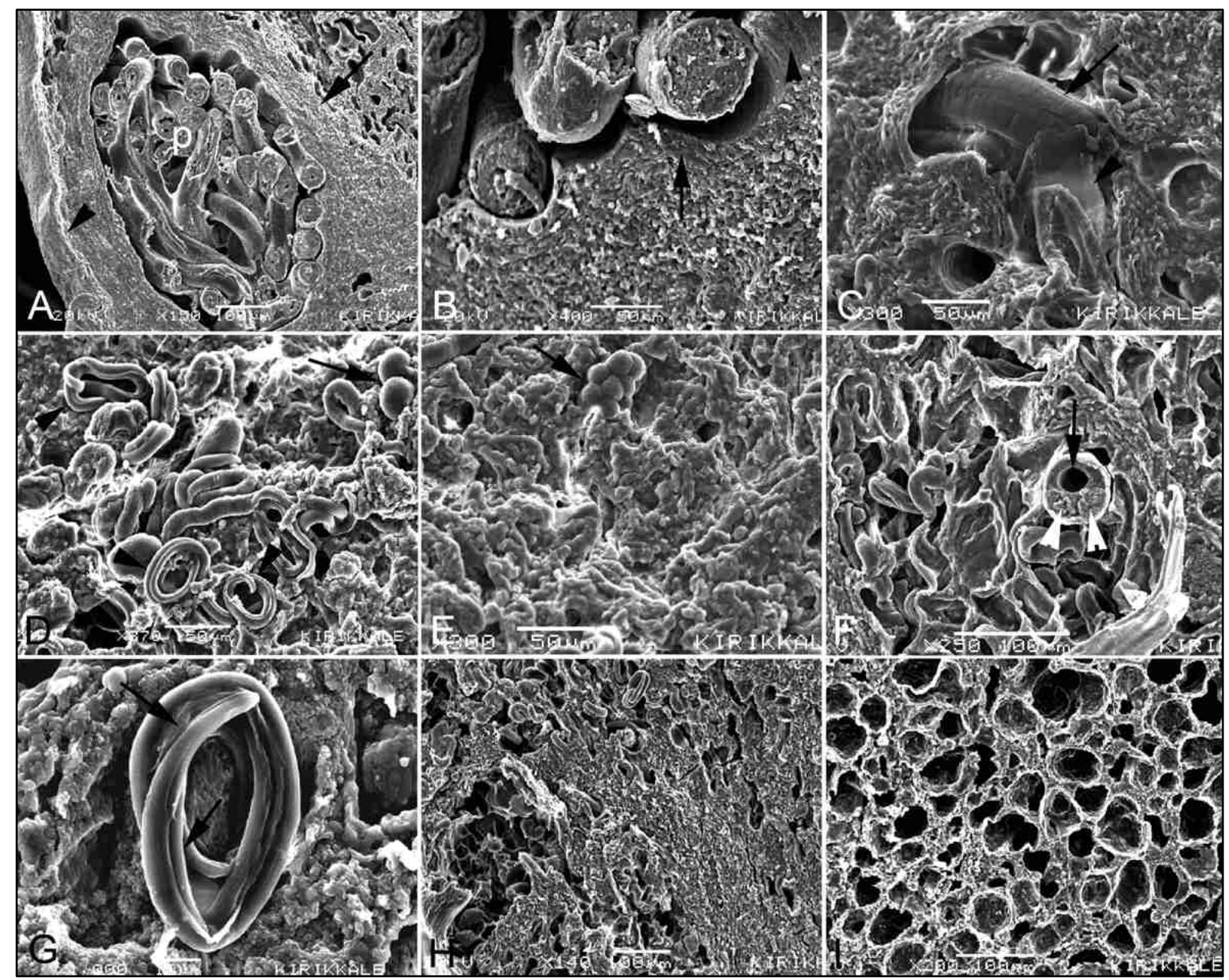

Fig. 1. Scanning electron micrograph of the lung with Cystocaulus ocreatus infection. A) In the worm nodule, the parasite (p) is enclosed by a capsule (arrow) located near the pleura (arrowhead). B) The inner surface of the capsule is smooth (arrowhead) and possess large indentations

caused by the curled up parasite (arrow). C) In brood nodules, the mature parasite is located in alveoli (arrow). The cuticle (arrowhead) is frequently peeled off artificially. D and E) The brood nodule also contains larvae (arrowhead) and eggs (arrow) at various stages of development in the vicinity of the mononuclear cell deposition. F) A cross section view of the mature parasite illustrating the digestive (arrow) and the reproductive truct (arrowheads). G) Larvae I curled up in eggshell (arrows). H) General view of the affected regions that is filled with parasites at different stages and mononuclear cell depositions. I) A view of healthy alveoli. 
ing to morphological characteristics of parasites (Levine, 1968).

\section{Results}

In SEM analysis, the worm nodule composed of a capsule enclosing the curled up immature parasite (Fig. 1A). The worm nodule was located adjacent to the lung surface; however, the pleura was distinguishable from the capsule. The capsule was very prominent with two distinct layers: the inner fibrous layer and the outer layer, which was composed mainly of mononuclear cells. The inner surface of the capsule was smooth (Fig. 1B). In regions of the lung where the brood nodules were located, the mature parasite was observed in alveoli (1C). The affected lung tissue was filled by larvae and different stages of eggs. These affected regions were also characterized by mononuclear cell depositions instead of normal alveoli (Fig. 1D and 1E). In the cross sections of the mature parasite, a circular well-defined intestine and reproductive tract were present (Fig. 1F). Although often stripped off during tissue processing, eggshell encapsulating larvae was observed in some instances (Fig. 1G). The regular lung morphology with well-defined alveoli no longer exists in the vicinity of brood nodules (Fig. 1H and 1I).

In light microscopic observation, the worm nodule was observed as it was localized closer to the lung surface (Fig. 2A). The cellular composition of the capsule became more apparent as the part closer to the inner capsule surface was more fibrous while the outer part was composed mainly of mononuclear cell depositions (Fig. 2B). The brood nodule was characterized by the presence of the mature parasite along with larvae and eggs at the different stages of development (Fig. 2C and 2D). In the affected lung regions, mononuclear cell infiltrations were also present beneath the bronchial epithelia (2E). In lung areas, which were devoid of brood or worm nodule, the interalveolar septa were highly hyperemic (Fig. 2F).

In SEM analysis of the parasite recovered from the worm nodule, the mouth was localized at the anterior tip of the parasite. Its diameter was relatively narrower compared to the body (Fig. 3A). A well-demarcated collar of tissue limited the mouth. A circle of tissue, with 6 papillae on it, was located just immediately behind the circular collar (Fig. 3A). Immediately behind the first line of papillae, another circle of papillae was positioned; however, they

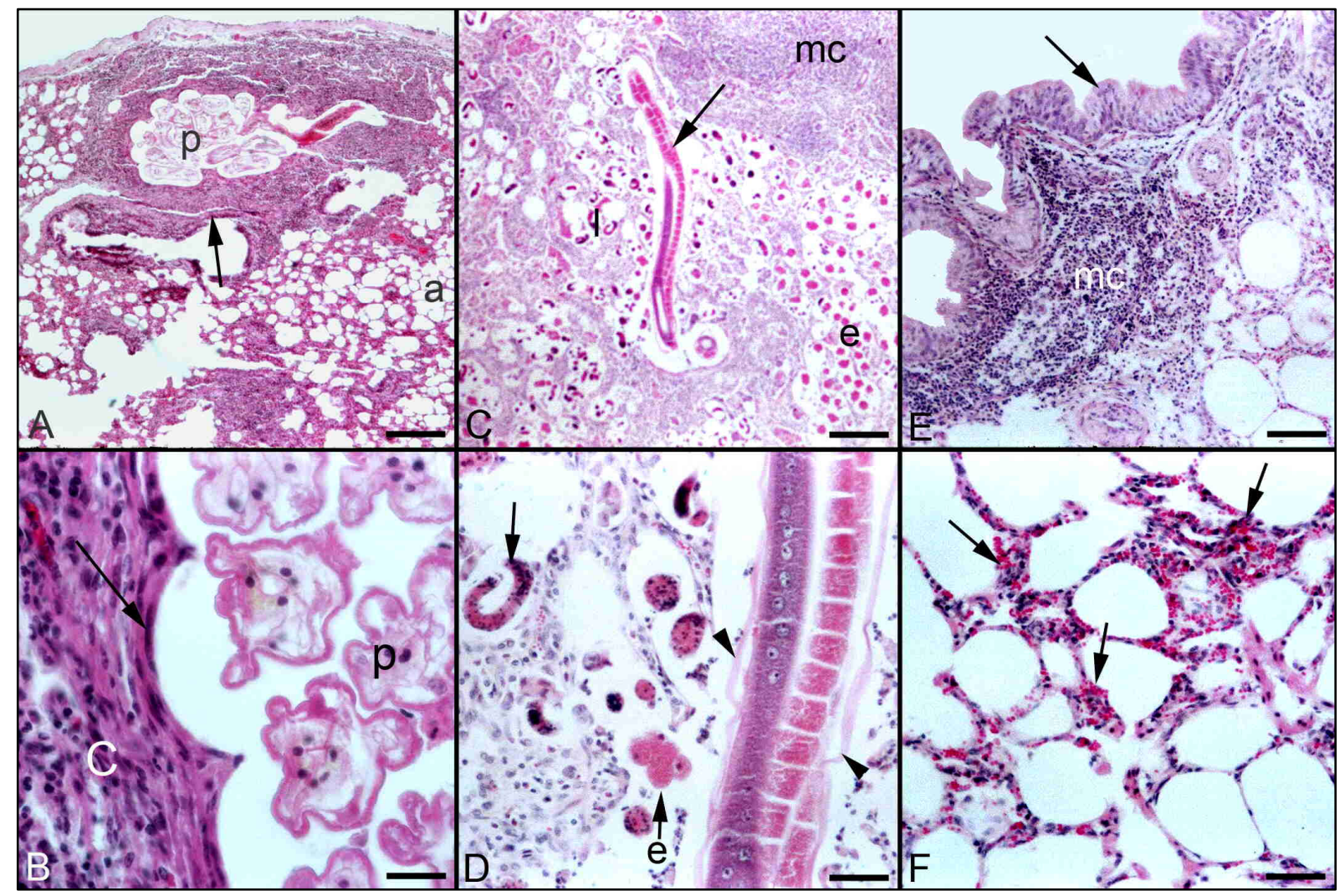

Fig. 2. Photomicrograph illustrating the light microscopic characteristics of the worm and brood nodules in the lung with Cystocaulus ocreatus infection. A) Worm nodules composed of a parasite (p) that is encapsulated by a capsule (arrow) a = alveoli. B) The portion of the capsule (c) adjacent to the inner surface (arrow) is fibrous in nature while the outer portion of the capsule is composed mainly of mononuclear cell deposition $\mathrm{p}=$ parasite. C) The brood nodule is composed of mature parasites (arrow), larvae (1), eggs (e) at different stages of development, and mononuclear cell infiltration (mc). D) The mature parasite has a well-defined cuticle (arrowheads) on the surface. Larvae (arrow); e = dividing egg. E) In infected lungs, mononuclear cell infiltration (mc) beneath the bronchial epithelia (arrow) is common. F) In affected lungs, interalveolar septa are highly hyperemic (arrows) in the region of the lung where parasite and its larvae and eggs are not present. Hematoxylin \& Eosin staining; bar = $125 \mu \mathrm{m}$ in $\mathrm{A}$ and $\mathrm{C}, 12.5 \mu \mathrm{m}$ in $\mathrm{B}, 25 \mu \mathrm{m}$ in $\mathrm{D}$ and $\mathrm{F}, 50 \mu \mathrm{m}$ in $\mathrm{E}$. 
were not as prominent as those in the first circle of papillae (Fig. 3A and 3B). In the neck, cuticular rings began just after the second circle of papillae (Fig. 3B). The width of the cuticular rings gradually increases from the neck towards the body (Fig. 3B and 3C). There were no sex differrences at the anterior end. There were longitudinal straight folds on the surface of the parasite that began behind of the second circle of papillae (Fig. 3B and 3C). Other than these folds and cuticles, no other specific modifications were observed in the surface of the body. At the posterior end, the female had a pro-vagina in the shape of a half bell, attaching to the other genital regions with folds of tissue (Fig. 3D and 3E). The surface of the pro-vagina was devoid of longitudinal folds so that it appears smoother (3E). The tail of the female was sharply pointed and a ring of tissue cuffs the posterior end just prior to the posterior tip
(Fig. 3E and 3F). The male posterior end had a bursa that housed the entire male genitalia (Fig. 3G). The rays on the bursa membrane were more prominent on the outer surface compared to inner surface (Fig. 3G). The anus was located at the very posterior end of the genitalia (Fig. 3G). Two spicules were not observed since they were retracted back inside to the anus. However, their positions were approximated with the prominent swellings in the anus (Fig. 3G and $3 \mathrm{H})$.

\section{Discussion}

Cystocaulus ocreatus belongs to Protostrongylidae family whose female lay down unembryonated eggs in the lung where the unembryonated eggs develop to the first stage larvae (Levine, 1968; Anderson, 2000). Using SEM along

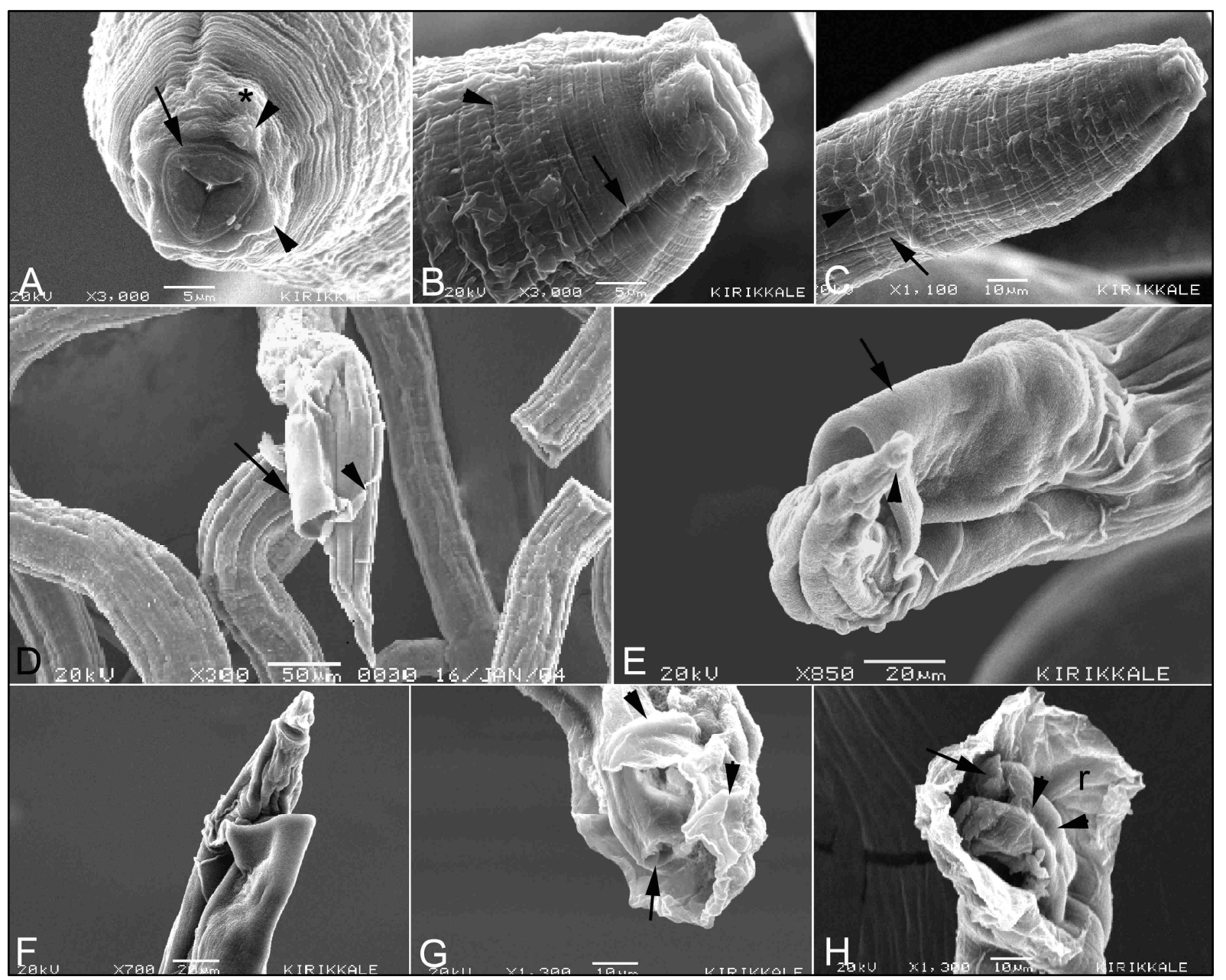

Fig. 3. Scanning electron micrograph of Cystocaulus ocreatus recovered from the worm nodule. A) The mouth is located at the anterior tip of the parasite. A collar of tissue (arrow) limits the mouth. A circle of tissue with 6 papillae (arrowheads) on it is located immediately behind the circular collar. Behind the first line of papillae, another circle of papillae $(*)$ not as prominent, was positioned. B and C). Cuticlar rings (arrowhead) begin just after the second circle of papillae. The width of the cuticlar rings gradually increases from the neck to the body. Initiating from the behind of the second circle of papillae, longitudinal straight folds (arrows) on the surface extend all the way to the posterior end. D) The female has a provagina (arrow) in the shape of a half bell that attached to the other genital region with folds (arrowhead). Note: the longitudinal irregular folding in this view is artifact. E and F) The pro-vagina (arrow) is devoid of folds so its surface is smoother. The tail of the female is sharply pointed, and a

tissue cuff encircles the posterior end just prior to the tip. G) A bursa that houses the anus (arrow) constitutes the posterior end. The rays (arrowheads) on the bursa membrane are more prominent on the outer surface. H) The tip of a spicules (arrow) is seen this picture. The positions of the spicules can be approximated with the prominent swellings (arrow heads) in the anus. $r=$ rays 
with light microscopy, we present the vivid views of dividing eggs at different stages of development that would yield to the first stage larvae, immature, and mature parasite in the lung.

Cystocaulus ocreatus infection results in lung lesions due not only to the presence of mature parasite but also its larvae and eggs as well as inflammatory reaction against them. Depending of the abundance of eggs, larvae, imamture and mature parasite as well as the severity of inflamematory reactions, gaseous exchange in the lung is interfered; therefore, pulmonary performance is reduced dramatically (Švarc, 1984; Berrag \& Cabarret, 1996). The functional loss of the lung especially where brood nodules were located can be easily perceptible from the three dimensional SEM pictures in the present study. In the regions of the lung where brood and worm nodules are present, almost no alveoli with normal morphology are present. Instead, mononuclear cell deposition and parasites along with their larvae and eggs occupy the area. These areas were described as airless areas by Švarc (1984) in chamois lungs infected by Muellerius capillaris, Muellerius tenuispiculatus and Neostrongylus linearis.

Histological examination of the lung lesions in infections by lung worm including $M$. capillaris, $M$. tenuispiculatus and $N$. linearis showed hyperplasia of peribronchial and perivascular lymphatic tissues (Švarc, 1984). Similarly, the peribronchial inflammatory cell depositions, mainly lymphocytic, in the present study were common.

Although the formation of brood and worm nodules of the C. ocreatus, it presumed that the immature C. ocreatus in worm nodules is encapsulated by a capsule which eventually may be destroyed and the parasite becomes free and then forms colonies that yield to brood nodules containing unembryonated eggs, the first stage larvae as well as female and male parasites (Schnieder, 2000). The SEM and light microscopy revealed that the capsule of the worm nodule is composed of two distinct layers: the inner layer which is more fibrous and the outer layer composed mainly of mononuclear cell deposition. It is questionable if the inflammatory mediators elaborated form the inflammatory cells in the outer layer help destroy the inner capsule. Therefore, the potential roles of the parasite inside the capsule and inflammatory cells should be further studied for the breakage of the capsule that may possibly lead to a differrent concept in intervention of $C$. ocreatus infections.

As cited by Berrag and Cabaret (1997), the worm and brood nodules correspond to nodular and lobular (diffuse) types of lesions, respectively in a different description. The gross lesions in M. capillaris, M. tenuispiculatus and $N$. linearis infection, Švarc (1984) describes the lesions as follows: petechiae, airless areas, indurated foci and breeding nodules. In our study, we just differentiated the brood and worm nodules formed by $C$. ocreatus.
In conclusion, the fine structure of morphology of $C$. ocreatus and the intact lung tissue infected with this parasite are presented in this study. The future studies should focus on the mobility of mature and immature parasites with respect to new understanding of structural integrity.

\section{Acknowledgements}

This project was financially supported by Research Fund of Kirikkale University (BAP-project No: 02/09-02-08).

\section{References}

ANDERSON, R. C. (2000): Nematode Parasites of Vertebrates. Their Development and Transmission. $2^{\text {nd }}$ Edition. CABI Publishing, CABI International.

Berrag, B., CABARret, J. (1996): Impaired pulmonary gas exchange in ewes naturally infected by small lungworms. Int. J. Parasitol., 26: 1397 - 1400

BERRAG, B., CABARRET, J. (1997): Assessment of the severity of natural infections of kids and adult goats by small lungworms (Protostrongylidae, Nematoda) using macroscopic lesion scores. Vet. Res., 28: 143 - 148

GuTTEKOVA, A., ZMORAY, I. (1974): Comparative study of the ultrastructure of muscle cells in pneumohelminths. Helminthologia, 15: $585-602$

Hernandez de Lujan, S., Muela de la, N., Ferre, I. (2001): Helminth parasites in goats in Valencia region. Med. Vet., 18: $476-482$

Levine, N. D. (1968): Nematode Parasites of Domestic Animals and of Man. Burgess Publishing Company, USA.

Meana, A., Luzon-Pena, M., SAntiago-Moreno, J., Bulnes, A. De, Gomez-Bautista, M., De Bulnes, A. (1996): Natural infection by gastrointestinal and bronchopulmonary nematodes in moufflons (Ovis musimon) and their response to netobimin treatment. J. Wildlife Dis., 32: $39-43$

Radfar, M. H., Ghorbani, A. (2003). A report on the helminth infections of native sheep slaughtered at Kerman abattoir. IJVR, 4: $109-113$

SATTLEROVÁ-ŠTEFAnČíKOVÁ, A. (2005): Chamois and its Parasites. Press Print, Košice

SCHNIEDER, T. (2000): Helminthosen der Wiederkauer. In Rommel, M., ECKert, J., Kutzer, E., Korting, W., SCHNEIDER, T. (Eds): Veterinarmedizinische Parasitologie. 5., Vollstandig neubearbeitere Auflage. Parey Buchverlag, Berlin. pp. $192-295$

ŠVARC, R. (1984). Pulmonary nematodes of the chamois Rupicapra rupicapra tatrica Blahout, 1971. 1. Pathomorphological picture of lungs during the development of worms into the adult stage. Helminthologia, 21: $141-150$ YILDIZ, K. (2006): Prevalence of lungworm infection in sheep and cattle in Kırıkkale province. T. Parazitol. Derg., 30: (In press) 